\title{
Evaluación de la degradabilidad in situ en bovinos suplementados con cuatro especies arbóreas
}

\section{Evaluation of in situ degradability in cattle supplemented on four tree species}

\author{
María Roa $V^{1}{ }^{1 *}$ M.Sc, Javier Muñoz M, ${ }^{2}$ MVZ. \\ ${ }^{1}$ Universidad de los Llanos, Escuela de Ciencias Animales Km 12 vía a Apiay, Villavicencio, Meta, \\ Colombia. ${ }^{2}$ Centro Educativo Rural calle16 No 1A-52, Restrepo, Meta, Colombia. *Correspondencia: \\ ligiaroa2607@gmail.com.
}

Recibido: Junio de 2010; Aceptado: Septiembre de 2011.

\section{RESUMEN}

Objetivo. Evaluar degradabilidad in situ en rumen de cuatro especies forrajeras: Acacia Roja (Delonix regia), pízamo (Eritryna glauca), Cratilia (Cratylia argentea) y casco de vaca (Bahuinia variegata), para determinar su calidad nutricional. Materiales y métodos. Cuatro hembras rumino-fistuladas en un diseño de sobrecambio simple, pastoreando en Brachiaria decumbens, suplementadas en la mañana con tres $\mathrm{kg}$ de hojas deshidratadas de las cuatro especies mencionadas, de un año de establecidas y podadas cada 3 meses. En las pruebas in situ se utilizaron bolsas de nylon, adicionando $5 \mathrm{~g}$ de MS de cada arbórea/bolsa, incluyendo braquiaria, en diferentes horas $(6,12$, 24, 48 y 72). Se evaluó la degradabilidad de la materia seca (DMS), fibra detergente neutro (DFDN) fibra detergente ácido (DFDA), nitrógeno total (DNT) y nitrógeno adherido a FDN (DNFDN). En el líquido ruminal se midió nitrógeno amoniacal a las $0,4,8$ y 12 y pH a las 0, 3, 6, 9 y 12 horas. Resultados. La DMS fue mayor $(p>0.05)$ para casco de vaca $(53.3 \%)$ y acacia roja $(56.1 \%)$ con relación a pízamo y cratilia. La DMS de braquiaria fue mayor ( $p>0.05$ ) en $18.6 \%$ suplementando con casco de vaca con relación a las otras arbóreas. La DFDN potencial fue menor $(p>0.05)$ para pízamo (7.6\%) en comparación con cratilia. La DFDN de braquiaria fue similar en todas las forrajeras. Conclusiones. Algunos componentes de las arbóreas tienen efecto asociativo en la cinética de la tasa de degradación de MS y FDA del pasto, siendo superiores $(p>0.05)$ cuando se suplementó con casco de vaca.

Palabras clave: Alimentación, follaje, rumiantes (Fuente:CAB). 


\section{ABSTRACT}

Objective. Evaluate the ruminal in situ degradability of four forage species: Flamboyant (Delonix regia), Indian Coral Tree (Erithryna glauca), Cratylia (Cratylia argentea) and Camel's Foot Tree (Bahuinia variegata) to determine their nutritional value. Materials and methods. Four rumenfistulated bovine females distributed in a simple crossover design, grazing on Brachiaria decumbens were supplemented in the morning with three $\mathrm{kg}$ of dried leaves of the four mentioned species. The tree species were one year old, and pruned every three months. Nylon bags with 5 grams of tree MS in each bag including $B$. decumbens were used for the in situ testing, at different times $(6,12,24,48$ and 72). The degradability of dry matter (DDM), neutral detergent fiber (DNDF), acid detergent fiber (DADF), total nitrogen (DTN) and nitrogen attached to FDN (DNANDF) were evaluated. Ammonia nitrogen was measured at $0,4,8$ and 12 and $\mathrm{pH}$ at $0,3,6,9$ and 12 hours was measured in the ruminal fluid. Results. The DMS was greater $(p>0.05)$ for $B$. variegata $(53.3 \%)$ and $(56.1 \%)$ for $D$. regia compared to E. glauca and C. argentea. The B. decumbens DMS was higher $(p>0.05)$ with $18.6 \%$ supplement of $\mathrm{B}$. variegata compared to the other trees. The potential DFDN was lower $(p>0.05)$ for $E$. glauca $(7.6 \%)$ compared with cratylia. The DFDN of $B$. decumbens was similar for all forages. Conclusions. Some components of the tree species have association effects on the kinetics of the the $B$. decumbens DM and ADF degradation rates, being higher $(p>0.05)$ when supplemented with $B$. variegata.

Key words: Fodder, food, ruminants (Source:CAB).

\section{INTRODUCCIÓN}

Los sistemas agroforestales tienen como objetivo central manejar de forma integrada especies arbóreas y actividades agropecuarias sin detrimento de los recursos naturales, por lo tanto,se recomienda utilizar árboles forrajeros en la producción ganadera. Los forrajes como las especies arbóreas contienen fibra, la cual es degradada por bacterias que producen la celulasa y que pertenecen al género Ruminococcus Bacteroides y Butyrivibrio, cuya población se puede ver afectada por factores como: tipo de dieta y forraje, tasa de pasaje, tamaño de partículas y otros. La fibra tiene como función mantener cercano a la neutralidad el $\mathrm{pH}$ del rumen lo que favorece el incremento de la población de las bacterias celulolíticas y por tanto, la disponibilidad de ácido propiónico que es la principal fuente de energía del rumiante (1-3).

Además, de la energía, es importante para la dieta del ganado las fuentes de proteína como son las leguminosas a las que pertenece la acacia roja (Delonix regia), también conocida como: flamboyant, flamboyán, flamboyanttree, flamboyán rojo y árbol de fuego por las flores de varios colores; se encuentra distribuido en todo el continente Americano. Además, de ser utilizada para ornamentación y sombrío se viene empleando para la alimentación de animales como lo demuestran investigaciones realizadas en México, donde en ciertas épocas el pasto para el ganado escasea, mientras que la acacia roja produce una alta cantidad de hojas y vainas, lo que la convierte en una alternativa forrajera para dichos periodos (4).

En Colombia, se ha venido investigando la acacia roja en cuanto a su uso como árbol forrajero en la alimentación de ganado. Se ha reportado que el contenido de proteína de sus hojas es del $17.0 \%$ y de fibra detergente neutra de $40.3 \%$, con una degradabilidad ruminal de la materia seca a las $72 \mathrm{~h}$ de $65.2 \%$, además, tiene gran aceptabilidad por parte del ganado y se adapta muy bien a las condiciones del Piedemonte Llanero $(5,6)$. Se realizaron análisis nutricionales en las hojas de: Pízamo (Erithryna glauca) y acacia roja (Delonix regia), cuyos resultados fueron: Proteína cruda (20.0 y $16.8 \%)$, FDN (55.7 y $28.0 \%)$, FDA (8.9 y $17.8 \%$ ) y digestibilidad in vitro de la MS (59.0 y $40.1 \%$ ), respectivamente. Se resalta que el pízamo tiene un mayor contenido nutricional en sus hojas, aunque la acacia se adapta mejor a condiciones críticas (7).

En trabajos con pízamo (Erithryna glauca) en bovinos fistulados se evaluó la degradabilidad ruminal de tres especies de árboles en las que se incluyó el pízamo; se encontró que la degradabilidad de la materia seca a las $72 \mathrm{~h}$ fue mayor para el Hibiscus rosa-sinensis (91.3\%) en comparación con las Erythrina glauca y $E$. poeppigiana: 44.3 y $52.0 \%$ respectivamente. En pízamo, la degradabilidad a las 72 h de la fibra detergente neutro fue de $27.0 \%$ y de nitrógeno total $59.0 \%$ (8). El nitrógeno en las primeras 
seis horas tiene una degradación rápida que llega al $33 \%$, siendo más lenta en las siguientes horas $[12,24,48$ y 72$](8,9)$.

El pízamo es una leguminosa que al ser sembrada en buenos suelos se puede realizar su primer corte a los 6 meses con podas posteriores entre 3 y 4 meses. Esta especie fue ofrecida a cabras y la producción de leche aumentó en $5 \%$ a medida que se incrementó la proporción de hojas de pízamo en una dieta básica de Kinggrass y banano de desecho. También se han observado buenos resultados al suministrarlo mezclado con los pastos como imperial (Axonopus scoparius) y elefante (Pennisetum purpureum) (9).

En investigaciones con cratilia (Cratylia argentea), se valoró la degradabilidad ruminal de la materia seca para comparar su composición nutricional y además, aprovechamiento, se observó que las composición química de la cratilia (Cratylia argentea) varía de acuerdo a la madurez y parte de la planta, reportando un contenido de proteína cruda de sus hojas entre el 11.0 y $17.0 \%$, con una digestibilidad in vitro de proteína entre el 42.0 y $44.0 \%$ (10).

Otras investigaciones con cratilia, utilizando vacas Holstein en pastoreo con Brachiaria decumbens se comparó con otro grupo que fue suplementado con ésta leguminosa en pastoreo restringido $2 \mathrm{~h}$ /día lo que incrementó la producción de leche y las cantidades de urea en éste producto y la sangre, los niveles de urea fluctuaron entre 14.9 y $18.7 \mathrm{mg} / \%$ vs 12.2 y $16.9 \mathrm{mg} / \%$ de grupo testigo. Las vacas permanecieron $50 \%$ del tiempo consumiendo leguminosa, estimando un periodo de pastoreo efectivo de media hora en la mañana y media en la tarde (11-13).

El casco de vaca (Bahunia variegata), es un árbol nativo de la India, se encuentra en todos los climas; puede llegar a una altura de 10 m (14), su nombre se debe porque sus hojas tienen forma del casco de una res. Se ha investigado los componentes químicos de esta especie, encontrando una sustancia similar a la insulina que se utiliza principalmente en la medicina para controlar los niveles de glucosa en la sangre. Actualmente, no solamentese se sigue utilizando para las personas con problemas de diabetes, sino para tratar enfermedades renales. Aunque este árbol es consumido por el ganado, es poco lo que se ha investigado para determinar su potencial forrajero $(14,15)$. El contenido de proteína es aproximadamente del $15.0 \%$, fibra detergente neutro $61.0 \%$ con una degradabilidad de la materia seca a las $72 \mathrm{~h}$ de $61.7 \%$ (5). En otros estudios, donde se hizo una caracterización bioquímica de las hojas de Bauhinia variegata, se encontró un alto valor proteínico y lipídico 29.41 y $14.89 \%$, respectivamente. También se observaron niveles elevados de: ácido linolénico y minerales, lo cual, la hace apta para ser usada como forraje, otros componentes detectados fueron: albúminas, globulinas, prolaminas, glutelinas ácidas y alcalinas, demostrado una actividad hipoglucimiante en conejos. Además del alto valor energético, de esta especie que le permite ser una fuente de alimentación, también desde el punto de vista farmacológico es una opción para el tratamiento de la diabetes y otras enfermedades cardiácas y renales (16).

Es importante tener en cuenta que en la degradabilidad de los forrajes influye su contenido de FDN, en la cual se incluye la lignina, este componente no es degradado por los microorganismos ruminales, es así que el proceso digestivo de los rumiantes, debe tener una adaptación para lograr una utilización eficaz de la pared celular de los forrajes, constituyendo estrategias digestivas considerablemente variables para cada especie, dependiendo de su de forma de cultivo y su fitogenética (12). La fibra además de producir energía para los rumiantes, es indispensable para preservar el equilibrio neurovegetativo, promoviendo el funcionamiento normal del tracto digestivo y regulando el tránsito de la ingesta, al mismo tiempo influye en la fermentación y mantenimiento de la integridad en la mucosa intestinal, previniendo los graves problemas digestivos (12).

El objetivo de esta investigación fue evaluar la degradabilidad in situ en bovinos fistulados de cuatro especies forrajeras: Acacia Roja (Delonix regia) pízamo (Eritryna glauca), Cratilia (Cratylia argentea), y casco de vaca (Bahunia variegata), estableciendo el efecto que tienen estas arbóreas en la eficiencia sobre la utilización ruminal de la pastura base con Brachiaria decumbens.

\section{MATERIALES Y METODOS}

Sitio de estudio. Esta investigación se realizó en Villavicencio, en la finca de UNILLANOS, sede Barcelona y en el Laboratorio de Nutrición Animal, ubicados en el Km 12 vía Puerto López en la Vereda Barcelona, con una altitud de 465 m.s.n.m. temperatura de $27^{\circ} \mathrm{C}$ y precipitación anual entre 1900 y 3250 milímetros. En esta zona se presentan cuatro meses de época seca (Diciembre a marzo) y ocho de lluvias. Las plantas, tenían un año de establecidas, y se podaron cada tres meses, sus hojas se secaron 
bajo techo para el suministro a los animales.

Tratamiento de hembras. Se utilizaron cuatro hembras criollas cruzadas, fistuladas en rumen con un peso vivo de $350 \pm 24.5 \mathrm{~kg}$, distribuidas en un diseño de sobrecambio simple con arreglo cuadrado latino (4X4). Las hembras fueron mantenidas en pastoreo continuo con braquiaria (Brachiaria decumbens), sal mineralizada y agua a voluntad. Los tratamientos fueron 3 kg de hojas secas suministradas en bateas de las siguientes especies: T1= acacia roja (Delonix regia), T2= pízamo (Erytrina glauca), $\mathrm{T} 3=$ cratilia (Cratilia argentea) y $\mathrm{T} 4=$ casco de vaca (Bahuinia variegata), para una mayor palatabilidad a éstas especies se les adicionó $300 \mathrm{~g}$ de melaza.

Periodos experimentales. Se establecieron cuatro periodos experimentales de 12 días cada uno, distribuidos así: los primeros 8 días fueron de adaptación a los forrajes en las pruebas de consumo previo y así lograr la aceptabilidad total de los tres $\mathrm{kg}$ de forraje ofrecido. En los otros cuatro días se colocaron las bolsas a incubar en el rumen para determinar su degradabilidad. En las pruebas in situ en rumen se utilizó la técnica de las bolsas de nylon $(17,18)$.

Bolsas. El tamaño de las bolsas fue de $20 \times 10$ $\mathrm{cm}$, con un poro promedio de 40 micras. Las bolsas se secaron previamente a $60^{\circ} \mathrm{C}$ por $24 \mathrm{~h}$ para llevarlas a peso constante y en cada una se colocaron $5 \mathrm{~g}$ de materia seca de forraje (arbóreas y pasto) por duplicado en diferentes horas $(6,12,24,48$ y 72), para observar el efecto de la eficiencia ruminal de los árboles sobre la dieta base. Con esta información se establecieron las degradabilidades de la materia seca (DMS), fibra detergente neutra, (DFDN) fibra detergente ácida (DFDA), nitrógeno total (DNT) y nitrógeno adherido a (DNFDN). En el líquido ruminal se realizaron mediciones de $\mathrm{pH}$ a las 0, 3, 6, 9, $12 \mathrm{~h}$ para establecer si se mantiene el pH normal (entre 5.5 a 6.8), el cual favorece el incremento de las bacterias celulolíticas y nitrógeno amoniacal a las 0, 4, 8 y $12 \mathrm{~h}$.

Forrajes secos. A los forrajes secos de: braquiaria, acacia roja, pízamo, cratilia y casco de vaca se les realizó un análisis nutricional preliminar, en el cual se determinó a cada uno materia seca (MS), extracto etéreo (EE), nitrógeno total (NT), cenizas, fibra cruda (FC), fibra detergente neutro (FDN), fibra detergente ácido (FDA) y extracto no nitrogenado (ENN), cuatro repeticiones por muestra con el fin de conocer la caracterización bromatológica de cada forraje (19-21).
Determinación de la tasa de degradación. Para la determinación de la tasa de degradación de la MS, FDN FDA, NT, NFDN, considerando el tiempo de incubación como variable independiente $(X)$ y las variables potencialmente digestibles $(Y)$, obteniéndose el coeficiente de regresión, aplicando el modelo matemático (22):

$$
Y=a+b\left(1-e^{-c^{*} t}\right)
$$

Donde:

$\mathrm{Y}=$ degradación potencial.

$\mathrm{t}=$ tiempo de incubación.

$\mathrm{a}=$ intercepto con el eje " $Y$ " en el tiempo cero. Representa el sustrato soluble completamente degradable que sale rápidamente de la bolsa de nylon.

$\mathrm{b}=$ Representa la fracción que se degradó a las $6,12,24,48$ y $72 \mathrm{~h}$.

$\mathrm{c}=$ tasa de degradación.

$\mathrm{e}=$ logaritmo natural

Diseño estadístico. Fue un sobrecambio simple con arreglo cuadrado latino $4 \times 4$, variables evaluadas: degradación ruminal $(6,12,24,48$ y 72 h) de la MS, FDN, FDA y NT, degradabilidad potencial aplicando modelo matemático (22). En el líquido ruminal se midió el $\mathrm{pH}$ y el nitrógeno amoniacal a las 0, 4, 8 y 12 h. Se realizó análisis de varianza, aplicando la prueba de Tukey con un nivel de confianza del 95\%, para comparación de medias. Se utilizó el programa estadístico SPSS 10 (23).

El Modelo estadístico aplicado fue:

$$
Y_{i j k}=\mu+T_{i}+f_{j}+c_{k}+\varepsilon_{i j k} \text { donde: }
$$

$Y_{i j k}$ es la lectura del tratamiento i-ésimo donde i= acacia roja, pízamo, cratilia y casco de vaca en la fila j-ésima, donde $j=$ periodo $1,2,3,4$ y en la k-ésima columna, donde $\mathrm{k}=$ animal fistulado $1,2,3,4$.

$\mu$ es la media general

$\mathrm{T}_{\mathrm{j}}$ es el efecto del tratamiento " $\mathrm{i}$ ", con $\mathrm{i}=1,2,3,4$.

$f_{j}$ es el efecto del periodo ' $j$ ", con $j=1,2,3,4$.

Ckes el efecto del animal fistulado"k", con k = $1,2,3,4$.

$\varepsilon_{i j k}$ es el error asociado con la lectura del i-ésimo tratamiento en el periodo j-ésimo y en el k-ésimo animal fistulado.

\section{RESULTADOS}

En la composición nutricional de los forrajes se observó que las especies arbóreas tienen entre 17.0 y $18.0 \%$ de proteína, lo cual indica que pueden ser un suplemento en la 
alimentación de ganado, puesto que el pasto braquiaria tiene el $6.6 \%$ de proteína. La FDN fue mayor en el pasto braquiaria $(67.8 \%)$ y la arbórea que mostró una FDN más alta fue casco de vaca $(58.2 \%)$. La FDA fue superior en pízamo (38.5\%) (Tabla1).

Tabla 1. Composición nutricional (\%). Promedio de cuatro repeticiones analizadas en cada forraje.

\begin{tabular}{lccccc}
\hline Nutrientes & Braquiaria & Acacia & Pízamo & Cratilia & $\begin{array}{c}\text { Casco } \\
\text { de vaca }\end{array}$ \\
\hline Materia & 27.3 & 35.0 & 26.8 & 31.8 & 41.7 \\
seca & \pm 0.9 & \pm 1.2 & \pm 0.9 & \pm 1.1 & \pm 1.4 \\
Proteína & 6.6 & 17.9 & 18.0 & 18.3 & 17.3 \\
& \pm 0.7 & \pm 0.6 & \pm 0.6 & \pm 0.6 & \pm 0.6 \\
Grasa & 2.1 & 3.5 & 4.3 & 2.7 & 5.7 \\
& \pm 0.3 & \pm 0.1 & \pm 0.1 & \pm 0.1 & \pm 0.2 \\
Fibra cruda & 37.0 & 19.3 & 28.0 & 18.9 & 19.2 \\
& \pm 1.4 & \pm 0.7 & \pm 0.9 & \pm 0.6 & \pm 0.6 \\
ENN & 41.6 & 48.3 & 3.9 & 51.9 & 47.8 \\
& \pm 1.5 & \pm 1.8 & \pm 0.6 & \pm 1.7 & \pm 1.6 \\
Cenizas & 4.6 & 8.5 & 37.8 & 5.7 & 7.0 \\
& \pm 0.4 & \pm 0.7 & \pm 1.3 & \pm 0.2 & \pm 0.3 \\
FDN & 67.8 & 57.5 & 51.1 & 49.2 & 58.2 \\
& \pm 2.6 & \pm 2.2 & \pm 1.7 & \pm 1.7 & \pm 2.0 \\
FDA & 32.5 & 30.2 & 38.5 & 32.7 & 36.7 \\
& \pm 1.2 & \pm 1.2 & \pm 1.3 & \pm 1.1 & \pm 1.4 \\
\hline
\end{tabular}

La degradabilidad materia seca (DMS) de la acacia fue superior $8.3 \%(p<0.05)$ en la mayoría de las horas en comparación con el pízamo y cratilia, pero a las $72 \mathrm{~h}$ la DMS de la acacia $(56.1 \%)$ y el casco de vaca $(53.0 \%)$ fueron similares y más altas $(p<0.05)$ en comparación con los otros dos tratamientos (Tabla 2).

Tabla 2. Degradabilidad de la materia seca (\%) en diferentes horas.

\begin{tabular}{ccccc}
\hline Horas & Acacia & Pízamo & Cratilia & Casco de vaca \\
\hline 6 & $34.2^{\mathrm{bc}}$ & $16.7^{\mathrm{a}}$ & $35.2^{\mathrm{c}}$ & $31.0^{\mathrm{b}}$ \\
12 & $38.9^{\mathrm{c}}$ & $20.6^{\mathrm{a}}$ & $38.0^{\mathrm{c}}$ & $35.3^{\mathrm{b}}$ \\
24 & $45.8^{\mathrm{b}}$ & $26.9^{\mathrm{a}}$ & $41.2^{\mathrm{b}}$ & $36.5^{\mathrm{b}}$ \\
48 & $51.0^{\mathrm{b}}$ & $44.6^{\mathrm{ab}}$ & $43.6^{\mathrm{a}}$ & $44.7^{\mathrm{ab}}$ \\
72 & $56.1^{\mathrm{b}}$ & $52.1^{\mathrm{a}}$ & $50.4^{\mathrm{a}}$ & $53.0^{\mathrm{ab}}$ \\
\hline
\end{tabular}

Letras distintas los tratamientos son diferentes $(p<0.05)$.

La DMS del braquiaria fue mayor $(p<0.05)$ con casco de vaca a las $24(54.5 \%), 48$ $(71.7 \%)$ y $72(77.1 \%)$ horas en comparación con: acacia $18.0 \%$, pízamo $23.0 \%$ y cratilia $18.0 \%$, la DMS del pasto a las 72 horas fue similar en las otras tres especies (Tabla 3 ).

La DFDN potencial de pízamo aplicando el modelo matemático, fue $66.3 \%$, valor inferior $(p<0.05)$ en comparación con acacia $(70 \%)$, cratilia $(76 \%)$ y casco $(73 \%)$, siendo similar
Tabla 3. Degradabilidad de la materia seca (\%) del pasto braquiaria.

\begin{tabular}{ccccc}
\hline Horas & Acacia & Pízamo & Cratilia & Casco de vaca \\
\hline 6 & $22.3^{\mathrm{bc}}$ & $19.0^{\mathrm{ab}}$ & $16.6^{\mathrm{a}}$ & $24.2^{\mathrm{c}}$ \\
12 & $29.0^{\mathrm{ab}}$ & $24.5^{\mathrm{ab}}$ & $21.0^{\mathrm{a}}$ & $30.1^{\mathrm{b}}$ \\
24 & $39.8^{\mathrm{b}}$ & $33.9^{\mathrm{a}}$ & $28.5^{\mathrm{a}}$ & $54.5^{\mathrm{c}}$ \\
48 & $49.7^{\mathrm{ab}}$ & $44.4^{\mathrm{a}}$ & $55.5^{\mathrm{b}}$ & $71.7^{\mathrm{c}}$ \\
72 & $58.8^{\mathrm{a}}$ & $55.9^{\mathrm{a}}$ & $60.8^{\mathrm{a}}$ & $77.1^{\mathrm{b}}$ \\
\multicolumn{4}{l}{}
\end{tabular}

esta variable en éstas tres últimas (Figura 1). La DFDN del pasto fue similar en todos los tratamientos, siendo en promedio mayor con cratilia en $2.1 \%$, lo que indica, que esta variable no se altera con el tipo de forraje (Tabla 4).

Tabla 4. Degradabilidad de la FDN Y FDA (\%) en diferentes horas de braquiaria.

\begin{tabular}{ccccc}
\hline Horas & Acacia & Pízamo & Cratilia & Casco de vaca \\
\hline $6^{*}$ & $12.5^{\mathrm{a}}$ & $14.0^{\mathrm{a}}$ & $14.6^{\mathrm{a}}$ & $22.9^{\mathrm{b}}$ \\
$12^{*}$ & $19.7^{\mathrm{ab}}$ & $13.7^{\mathrm{a}}$ & $24.0^{\mathrm{b}}$ & $39.1^{\mathrm{c}}$ \\
$2^{*}$ & $34.2^{\mathrm{b}}$ & $21.6^{\mathrm{a}}$ & $47.8^{\mathrm{c}}$ & $50.1^{\mathrm{c}}$ \\
$48^{*}$ & $51^{\mathrm{b}}$ & $36.8^{\mathrm{a}}$ & $59.3^{\mathrm{b}}$ & $54.5^{\mathrm{b}}$ \\
$72^{*}$ & $62.9^{\mathrm{a}}$ & $58.7^{\mathrm{a}}$ & $63.0^{\mathrm{a}}$ & $61.0^{\mathrm{a}}$ \\
$6^{* *}$ & $15.2^{\mathrm{a}}$ & $19.2^{\mathrm{a}}$ & $24.8^{\mathrm{b}}$ & $25.8^{\mathrm{b}}$ \\
$12^{* *}$ & $19.7^{\mathrm{a}}$ & $24.4^{\mathrm{b}}$ & $30.6^{\mathrm{c}}$ & $26.3^{\mathrm{b}}$ \\
$24^{* *}$ & $25.7^{\mathrm{a}}$ & $32.7^{\mathrm{b}}$ & $30.3^{\mathrm{b}}$ & $37.0^{\mathrm{c}}$ \\
$48^{* *}$ & $32.8^{\mathrm{a}}$ & $40.4^{\mathrm{b}}$ & $38.4^{\mathrm{ab}}$ & $43.7^{\mathrm{bc}}$ \\
$72^{* *}$ & $42.5^{\mathrm{a}}$ & $44.1^{\mathrm{a}}$ & $51.8^{\mathrm{b}}$ & $54.3^{\mathrm{b}}$ \\
\hline
\end{tabular}

Letras distintas los tratamientos son diferentes $(p<0.05)$.

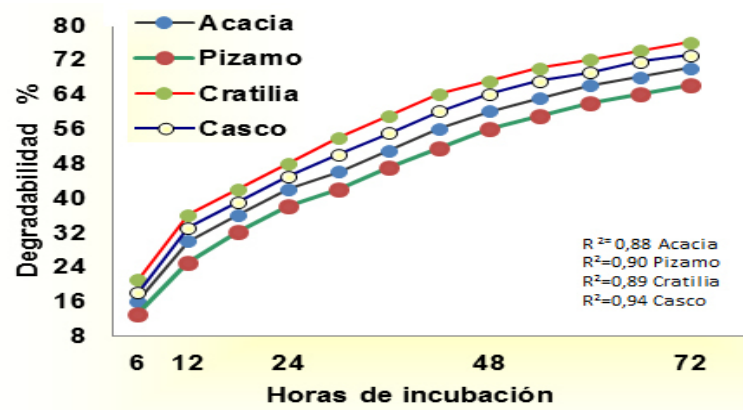

Figura1. Degradabilidad potencial de la FDN.

La DFDN potencial en todos los tratamientos fue mayor que la DFDA, siendo ésta última menor $(p<0.05)$ para pízamo en $3.6 \%$ con relación a las otras tres especies (Figura 2). La DFDA efectiva del pasto braquiaria fue más alta $(p<0.05)$ con casco de vaca $(54.3 \%)$ y cratilia $(51.8 \%)$ en comparación con acacia $(42.5 \%)$ y pízamo $(44.1 \%)$, lo que indica que la gradación de la lignina y la celulosa del pasto está influenciada por la composición del árbol (Tabla 4). La DNT potencial fue inferior 


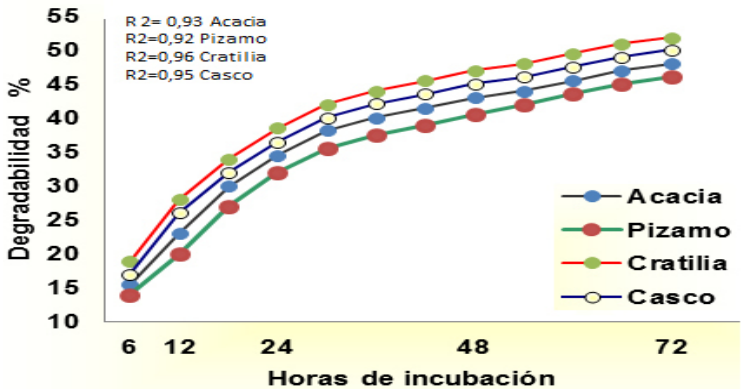

Figura 2. Degradabilidad potencial de la FDA.

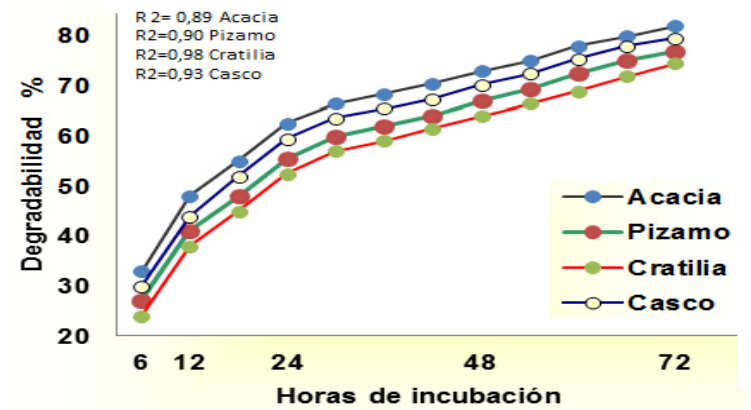

Figura 3. Degradabilidad potencial del NT.

$(\mathrm{p}<0.05)$ para cratilia $(74.5 \%)$ con relación a los demás tratamientos: acacia (82.0\%), pízamo $(77.0 \%)$ y casco $(79.6 \%)$ (Figura 3 ). La DNT del pasto fue superior $(p>0.05)$ en casco de vaca, con relación a: cratilia (31.9\%) acacia ( $22.4 \%$ ) y pízamo $(22.1 \%$ ) (Tabla 5 ).

La DNFDN fue similar $(p>0.05)$ en todas las especies: acacia $(58.0 \%)$, pízamo $(60.5 \%)$, cratilia $(62.0 \%)$ y casco $(57.0 \%)$ (Figura 4$)$. La DNFDN del pasto braquiaria fue mayor en $4.6 \% \quad(p<0.05)$ en pízamo en comparación con casco de vaca; con las otras arbóreas, esta variable fue similar (Tabla 5). La tasa de

Tabla 5. Degradabilidad de NT y NDFN (\%) en diferentes horas de braquiaria.

\begin{tabular}{ccccc}
\hline Horas & Acacia & Pízamo & Cratilia & Casco de vaca \\
\hline NT & & & & \\
6 & $24.0^{\mathrm{a}}$ & $28.5^{\mathrm{a}}$ & $24.0^{\mathrm{a}}$ & $26.3^{\mathrm{a}}$ \\
12 & $43.8^{\mathrm{c}}$ & $39.4^{\mathrm{bc}}$ & $30.7^{\mathrm{a}}$ & $37.8^{\mathrm{ab}}$ \\
24 & $46.6^{\mathrm{a}}$ & $48.9^{\mathrm{a}}$ & $49.6^{\mathrm{a}}$ & $50.9^{\mathrm{a}}$ \\
48 & $57.3^{\mathrm{b}}$ & $61.8^{\mathrm{bc}}$ & $50.1^{\mathrm{a}}$ & $64.7^{\mathrm{c}}$ \\
72 & $64.5^{\mathrm{b}}$ & $64.2^{\mathrm{b}}$ & $54.7^{\mathrm{a}}$ & $86.6^{\mathrm{c}}$ \\
NDFN & & & & \\
6 & $9.6^{\mathrm{a}}$ & $14.4^{\mathrm{ab}}$ & $24.3^{\mathrm{b}}$ & $17.9^{\mathrm{b}}$ \\
12 & $17.5^{\mathrm{a}}$ & $37.3^{\mathrm{b}}$ & $27.4^{\mathrm{ab}}$ & $25.6^{\mathrm{ab}}$ \\
24 & $44.9^{\mathrm{a}}$ & $55.9^{\mathrm{b}}$ & $39.7^{\mathrm{a}}$ & $41.1^{\mathrm{a}}$ \\
48 & $56.7^{\mathrm{ab}}$ & $61.3^{\mathrm{bc}}$ & $64.1^{\mathrm{c}}$ & $52.4^{\mathrm{a}}$ \\
72 & $64.4^{\mathrm{ab}}$ & $66.5^{\mathrm{b}}$ & $64.9^{\mathrm{ab}}$ & $61.9^{\mathrm{a}}$ \\
\hline Letras distintas los tratamientos son diferentes $(\mathrm{p}<0.05)$.
\end{tabular}

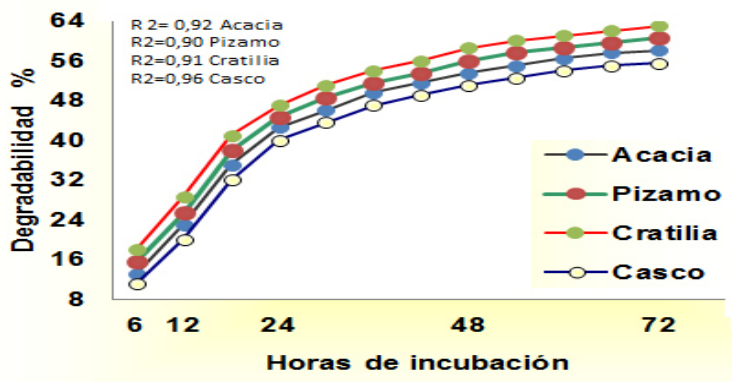

Figura 4. Degradabilidad potencial de la NFDN.

degradación (\% de forraje/hora) de la MS $(0.70)$ y NT (1.0) fueron menores $(p>0.05)$ en cratilia comparándola con las demás especies, (Tabla 6). La tasa de degradación de la FDN y FDA fueron menores $(p>0.05)$ para pízamo y acacia. La tasa de degradación (\% de forraje/hora) de la MS (1.1) y FDA $(0.86)$ del pasto fueron superiores $(p>0.05)$ para casco de vaca, mientras, la tasa de FDN de acacia pízamo y casco de vaca observaron similar comportamiento (Tabla 6).

Tabla 6. Tasa efectiva de degradación (\% de forraje/ hora).

\begin{tabular}{lcccc}
\hline \multicolumn{1}{c}{ Nutriente } & Acacia & Pízamo & Cratilia & Casco de vaca \\
\hline Materia Seca & $0.78^{\mathrm{b}}$ & $0.73^{\mathrm{ab}}$ & $0.70^{\mathrm{a}}$ & $0.74^{\mathrm{ab}}$ \\
FDN & $0.97^{\mathrm{ab}}$ & $0.92^{\mathrm{a}}$ & $1.1^{\mathrm{b}}$ & $1.0^{\mathrm{b}}$ \\
FDA & $0.66^{\mathrm{a}}$ & $0.64^{\mathrm{a}}$ & $0.72^{\mathrm{b}}$ & $0.69^{\mathrm{a}}$ \\
Nitrógeno total & $1.1^{\mathrm{b}}$ & $1.1^{\mathrm{b}}$ & $1.0^{\mathrm{a}}$ & $1.1^{\mathrm{b}}$ \\
NFDN & $0.80^{\mathrm{ab}}$ & $0.84^{\mathrm{ab}}$ & $0.88^{\mathrm{bc}}$ & $0.77^{\mathrm{a}}$ \\
Materia seca Pasto & $0.82^{\mathrm{b}}$ & $0.78^{\mathrm{a}}$ & $0.84^{\mathrm{b}}$ & $1.1^{\mathrm{c}}$ \\
FDN pasto & $0.87^{\mathrm{b}}$ & $0.81^{\mathrm{ab}}$ & $0.88^{\mathrm{b}}$ & $0.85^{\mathrm{ab}}$ \\
FDA pasto & $0.59^{\mathrm{a}}$ & $0.61^{\mathrm{a}}$ & $0.72^{\mathrm{c}}$ & $0.86^{\mathrm{d}}$ \\
\hline
\end{tabular}

Letras distintas los tratamientos son diferentes $(p<0.05)$.

Tabla 7. Nitrógeno amoniacal (NH3-N) (mg/100ml de líquido ruminal) y $\mathrm{pH}$ en líquido ruminal.

\begin{tabular}{ccccc}
\hline Horas & Acacia & Pízamo & Cratilia & Casco de vaca \\
\hline NH3-N & & & & \\
0 & $23.4^{\mathrm{ab}}$ & $22.5^{\mathrm{ab}}$ & $13.7^{\mathrm{a}}$ & $26.1^{\mathrm{b}}$ \\
4 & $23.8^{\mathrm{a}}$ & $23.0^{\mathrm{a}}$ & $16.8^{\mathrm{a}}$ & $15.9^{\mathrm{a}}$ \\
8 & $21.8^{\mathrm{a}}$ & $20.3^{\mathrm{a}}$ & $17.3^{\mathrm{a}}$ & $21.6^{\mathrm{a}}$ \\
12 & $18.5^{\mathrm{a}}$ & $19.3^{\mathrm{a}}$ & $17.2^{\mathrm{a}}$ & $17.6^{\mathrm{a}}$ \\
$\mathbf{p H}$ & & & & \\
0 & $6.5^{\mathrm{a}}$ & $6.6^{\mathrm{a}}$ & $5.9^{\mathrm{a}}$ & $7.4^{\mathrm{b}}$ \\
3 & $6.9^{\mathrm{ab}}$ & $6.8^{\mathrm{a}}$ & $6.8^{\mathrm{a}}$ & $7.3^{\mathrm{b}}$ \\
6 & $7.1^{\mathrm{b}}$ & $7.2^{\mathrm{b}}$ & $6.8^{\mathrm{a}}$ & $7.0^{\mathrm{ab}}$ \\
9 & $6.9^{\mathrm{ab}}$ & $7.1^{\mathrm{ab}}$ & $6.6^{\mathrm{a}}$ & $7.4^{\mathrm{b}}$ \\
12 & $6.8^{\mathrm{a}}$ & $7.0^{\mathrm{ab}}$ & $6.8^{\mathrm{a}}$ & $7.3^{\mathrm{b}}$ \\
\hline
\end{tabular}

Letras distintas los tratamientos son diferentes $(p<0.05)$. 
El $\mathrm{pH}$ a las $12 \mathrm{~h}$ fue mayor $(\mathrm{p}<0.05)$ para casco de vaca (7.3), mientras el nitrógeno amoniacal a las 12 horas fue similar para todos los tratamientos (entre 17.2 a 19.3 $\mathrm{mg} / 100 \mathrm{ml}$ de líquido ruminal) (Tabla 7 ).

\section{DISCUSIÓN}

Ordoñez y Diaz (9) encontraron para el pízamo porcentajes de FDN, FDA, DMS inferiores a los hallados en este trabajo en $1.8 \%, 23.1 \%$ y $12.9 \%$ respectivamente, mientras la proteína del pízamo fue en unidades porcentuales $2 \%$ inferior en comparación a la analizada por Narváez (4) (20.0\% vs $18.0 \%$ ), lo cual indica que los suelos y la forma de cultivar influyen en el contenido nutricional de los forrajes, puesto que suelos del Valle del Cauca son diferentes a los del Piedemonte llanero, donde las condiciones hacen que las plantas desarrollen más fibra. A pesar de esta situación se observa una mejor DMS con relación a la encontrada por este autor (4). También es de resaltar que en la acacia fue mayor el porcentaje de proteína $(17.9 \%$ vs $16.8 \%)$ comparándolas con el trabajo de Narváez y Lazcano (7). Evaluaciones con pízamo mostraron que fueron inferiores en unidades porcentuales: MS $(11.7 \%)$, FDN $(13.8 \%)$ y NT $(2 \%)$ a las encontradas en esta investigación (9). Se deduce que el tipo de metodología y la forma de tratamiento del cultivo de esta especie influye en los resultados de estas variables.

La DNT potencial de cratilia se observó en este trabajo que fue superior en $30 \%$ a la evaluada en forma in vitro (10), resultados que confirman lo expresado anteriormente, que los parámetros nutricionales pueden variar por el medio ambiente donde se establece la especies forrajera (10). Los análisis indicaron que la composición de las arbóreas está relacionada con la cinética de degradación del pasto, puesto que la tasa de degradación de la MS fue mayor con el casco de vaca y menor que con pízamo lo que concuerda con Ibrahim et al (12), con relación a que las mezclas de forrajes pueden ocasionar efectos asociativos que modifican la degradación de los nutrientes de otros forrajes. En este caso el pasto braquiaria incrementó su DMS $(p<0.05)$ cuando se suministró con casco de vaca (Tabla 3 ).

En la cratilia se observó una mayor FDN, FDA, y NFDN potencial, con relación a las otras especies (Figuras 1, 2 y 4) lo que implica que las bacterias del rumen fueron eficientes para degradar su pared celular y hacer disponible el nitrógeno adherido, lo cual beneficia el crecimiento de microorganismos.

La DNT potencial fue mayor con referencia a las otras arbóreas (Figura 3), lo que implica que los aminoácidos de esta leguminosa se convierten en alta proporción en nitrógeno para la población ruminal, disminuyendo la fracción de proteína sobrepasante.

En conclusión, la proteína de las especies estudiadas fue similar, $(18.0 \%)$, lo cual es ventajoso utilizarlas como suplemento para ganado en pastoreo con braquiaria $(6.6 \%)$. La DMS de acacia roja fue superior en comparación con las otras forrajeras, pero la DMS de pasto braquiaria es mejor cuando se suministra casco de vaca.

El comportamiento de la DFDN y DFDA potencial en la cinética del rumen es similar, siguiendo el orden de mayor a menor: cratilia, casco de vaca, acacia y pízamo. Se recomienda realizar pruebas de consumo utilizando bovinos suplementados con estas arbóreas, que de acuerdo con los resultados obtenidos pueden mejorar su capacidad productiva.

\section{REFERENCIAS}

1. Moallem $\mathrm{U}$, Lehrer $\mathrm{H}$, Livshitz $\mathrm{L}$, Zachut $M$, Yakoby $S$. The effects of live yeast supplementation to dairy cows during the hot season on production, feed efficiency and digestibility. J Dairy Sci 2009; 92:343-351.

2. García DE, Medina MG, Humbría J, Domínguez CE, Baldizán A, Cova LJ, Soca M. Composición proximal, niveles de metabolitos secundarios y valor nutritivo del follaje de algunosárboles forrajeros tropicales. Arch Zootecnia 2006; 55(212):373-384.
3. Marden JP, Julien C, Monteils V, Auclair $E_{\text {, }}$ Moncoulon R, Bayourthe $\mathrm{C}$. how does live yeast differ from sodium bicarbonate to stabilize ruminal ph in high-yielding dairy cows. J Dairy Sci 2008; 91:1320-1331.

4. Narváez N. Magnitud y tasa de fermentación in vitro de la materia seca y degradación de la fibra en diferentes especies arbóreas tropicales con uso potencial como forraje en Colombia. [Tesis de Maestría]. Palmira, Colombia: Universidad Nacional, Facultad de de ciencias Agropecuarias, Programa de Zootecnia; 2000. 
5. Roa ML, Céspedes DA, Galeano JR Muñoz J, Muñoz R. Utilización de árboles forrajeros para la alimentación de ganado en el Pie de Monte Llanero. [Trabajo de investigación]. Villavicencio, Colombia: Universidad de los Llanos, Facultad de Ciencias Agropecuarias y Recursos Naturales; 2000.

6. Roa ML. Contenido nutricional de forrajes y alimentos para animales. Villavicencio, Colombia: Universidad de los Llanos, Escuela de Medicinas veterinaria y Zootecnia; 2004.

7. Narváez N y Lascano C. Caracterización química de especies arbóreastropicales con potencial forrajero en Colombia. Pasturas Tropicales 2004; 26:1-8.

8. Roa M, Galeano J, Muñoz J. suplementación de vacas doble propósito con Morus alba Erythrina glauca en época seca en la subregión del pie de monte llanero Colombia. Rev Orinoquia 2002; 6:70-87.

9. Ordoñez O, Díaz G. Valor nutricional de forraje de pízamo (Erythrina glauca) ensilado con diferentes niveles de melaza y harina de arroz. [Trabajo de grado]. Villavicencio, Colombia: Universidad de los llanos, Programa de Medicina Veterinaria y Zootecnia, Facultad de Ciencias Agropecuarias y Recursos Naturales; 2002.

10. Lascano $\mathrm{C}$, Rincón A, Plazas $\mathrm{CH}$, Bueno G, Argel P. Cultivar veranera Cratylia argentea (desvaux) o. Kuntze. Leguminosa arbustiva de usos múltiples para zonas con períodos prolongados de sequia en Colombia. Cali,Colombia: CORPOICA, MINAGRICULTURA Y CIAT; 2002.

11. Aparicio R, Lascano C, Ávila P. Utilización de la Cratylia argentea madura y joven como banco de proteína por vacas de leche. Rev Científica 2002; 12:595-598.

12. Ibrahim M, Franco M, Pezo DA, Camero A, Araya JL. Degradabilidad ruminal in situ y solubilidad de la proteina de rebrotes de Cratylia argentea de diferentes edades. Deposito de documentos FAO [internet]. 2000 [Consulta 05 de septiembre de 2010]. disponible: En http://www.fao.org/wairdocs/LEAD/X6319S/ X6319S00.HTM.

13. Krause KM, Combs DK, Beauchemin KA. Effects of increasing levels of refined cornstarch in the diet of lactating dairy cows on performance and ruminal pH. J Dairy Sci 2003 86:1341-1353.
14. Jiménez I, Tovar MA. Evaluación de la producción de materia seca y valor nutritivo de (Cratylia argentea (desv.) O. Kuntze) a dos edades y dos alturas de corte en época seca y de lluvias en la finca Altamonte, municipio de Sahagún, departamento de Córdoba. [Tesis Zootecnista], Sincelejo, Colombia: Universidad de Sucre, Facultad de Ciencias Agropecuarias; Departamento de Zootecnia; 2003.

15. De los Ríos C, Gil H, Hidalgo D. Bauhinia variegata L. sobre la glucación no enzimática dela hemoglobina. Rev Fac Farm 2003; 45:12-16.

16. Da-Silva KL. Phytochemical y la investigación de pharmacognositc del Eslabón de forficata de Bauhinia. Z Naturforsch 2000; 55:478-480.

17. Pinto $L$, Andrade $M$, Bacarin $M$, Castellón R, Gadelha T, Gadelha C, Cavada B. Caracterização química e bioquímica de sementes de Bauhinia variegata L. Rev Bras Eng Agríc Ambient 2005; 9:1-9.

18. Godden SM, Lissemore KD, Kelton DF, Lumsden JH, Leslie KE, Walton JS. Analytic validation of an infrared milk urea assay and effects of sample acquisition factors on milk urea results. J Dairy Sci 2000; 83:435-442.

19. Mertens DR. Gravimetric determination of amylase-treated neutral detergent fiber in feeds with refluxing in beakers or crucibles: collaborative study. Journal of AOAC International. 2002; 85(6): 1217-1240.

20. AOAC. Official Methods of Analysis (18th) Arligton, VA. Washington, D.C: Association of Official Analytical Chemists; 2006.

21. Wilkins RJ. Forage Evaluation in Ruminant. Givens RFE, HM Omed (Eds.). Wallingford, Oxon: CAB International; 2000.

22. Rosero R, Posada S. Modelación de la cinética de degradación de alimentos para rumiantes. Rev Col Cienc Pec 2007; 20:174-182.

23. Alvarez C. SPSS 10 (programas para computador) | estadistica spss 10 para windows (programas para computador) |Análisis de datos [CD-Rom]. Madrid: Unversidad Complutense; 2001. 